\title{
Direct Detection of Optical Forces of Magnetic Nature in Dielectric Nanoantennas
}

\author{
Martin Poblet, Yi Li, Emiliano Cortés, Stefan A. Maier,* Gustavo Grinblat,* and Andrea V. Bragas \\ Cite This: Nano Lett. 2020, 20, 7627-7634 \\ Read Online
}

ABSTRACT: Optical forces on nanostructures are usually characterized by their interaction with the electric field component of the light wave, given that most materials present negligible magnetic response at optical frequencies. This is not the case however of a high-refractive-index dielectric nanoantenna, which has been recently shown to efficiently support both electric and magnetic optical modes. In this work, we use a photoinduced force microscopy configuration to measure optically induced forces produced by a germanium nanoantenna on a surrounding silicon near-field probe. We reveal the spatial distribution, character, and magnitude of the generated forces when exciting the nanoantenna at its anapole state condition. We retrieve optical force maps showing values of up to $20 \mathrm{pN}$, which are found to be mainly magnetic in nature, according

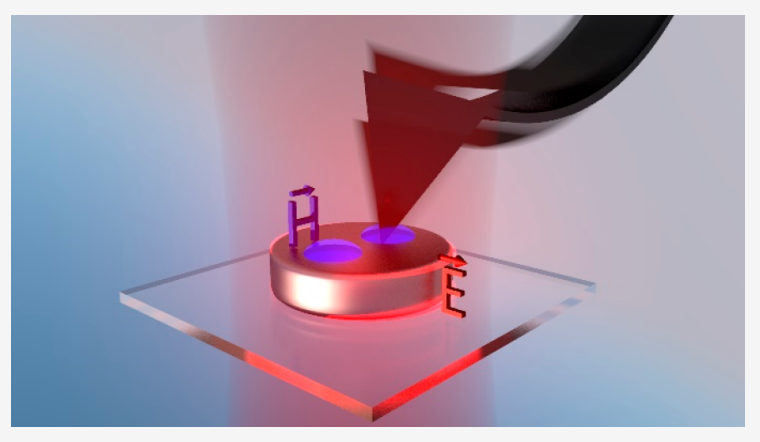
to our numerical simulations. The results of this investigation open new pathways for the study, detection, and generation of magnetic light forces at the nanometer scale.

KEYWORDS: Photoinduced Force Microscopy, Dielectric nanoantenna, Anapole excitation, Magnetic field enhancement

$\mathrm{L}$ ight carries energy as well as linear and angular momentum that can be transferred to atoms, molecules, and particles. The general law for electromagnetic forces relies on the conservation of momentum and connects the electromagnetic and the mechanical realms. Optical forces have been of enormous significance in describing a wide variety of fundamental physical phenomena with experimental realizations including milestones such as optical tweezers, ${ }^{1,2}$ laser printing of nanoparticles, ${ }^{3,4}$ ion trapping and cooling, ${ }^{5}$ and cavity optomechanics. ${ }^{6-8}$ In general, since most structures have a very weak magnetic response at optical frequencies, it is only the effect of the electric field of light on the optically induced forces that is usually considered. This is the case, for example, for a plasmonic nanoparticle, which can highly concentrate the electric field component of light at subwavelength volumes but cannot significantly enhance the magnetic component, because of the vanishing field inside the metal. ${ }^{9}$ Indeed, it was not until 2009 that the magnitude and vector character of the magnetic field of light in the near field was measured using a near-field probe with a split ring resonator design. ${ }^{10}$ Moreover, only very recently an algorithm that extracts all six components of the electromagnetic field with just a single near-field measurement has been published. ${ }^{11}$ In this context, enhancing the nanoscale magnetic response at optical frequencies could aid in completing our understanding of light-matter interactions in the same way that plasmonic systems have shed light on nanoscale manipulation of the electric component of light.
Different from the case of plasmonics, high-refractive-index dielectric nanoparticles have been recently shown to efficiently support both electric and magnetic optical modes through Mie resonances. ${ }^{9-15}$ Moreover, it has been proven experimentally that when a silicon nanoparticle is subjected to an intense light field, magnetic forces produce detectable effects. ${ }^{12}$ However, in general terms, the magnetic properties of optical nanoresonators have been only weakly characterized and utilized so far compared to their electric counterpart. Having a system supporting magnetic light resonances makes the dielectric nanoantenna a very attractive platform to measure optical forces of magnetic origin, in a similar way that optically induced electric forces have been detected close to metallic nanoparticles with photoinduced force microscopy (PiFM) ${ }^{13,14}$ PiFM combines optical microscopy with atomic force microscopy (AFM) $)^{15,16}$ to map electromagnetic nearfield forces on the order of femtonewtons to piconewtons using a polarizable probe.

Dielectric nanoparticles can be described by a complex electrical and magnetic polarizability and are expected to feel (and produce) electric and magnetic forces when illuminated

Received: July 31, 2020

Revised: September 15, 2020

Published: September 16, 2020 


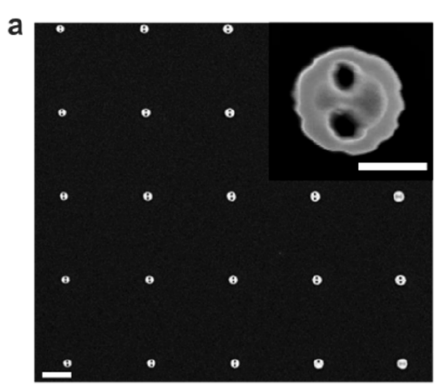

d

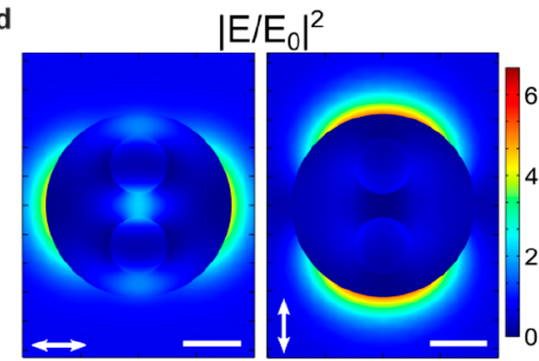

f

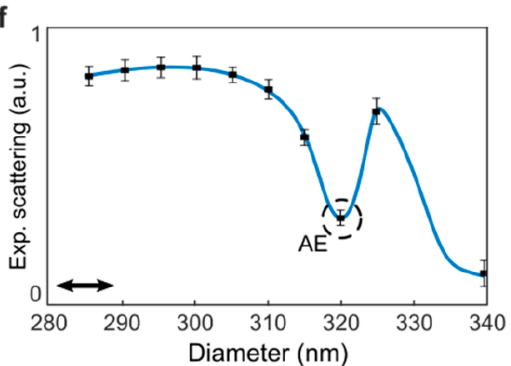

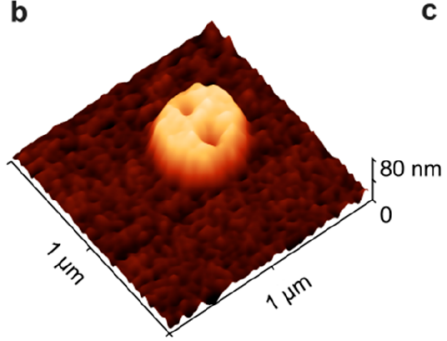

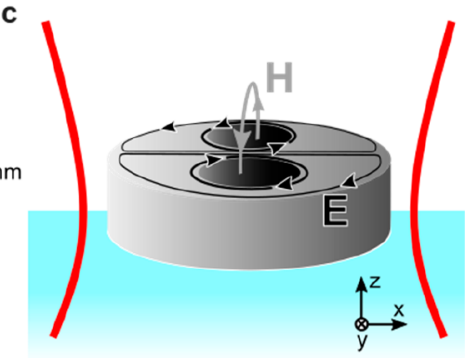

e

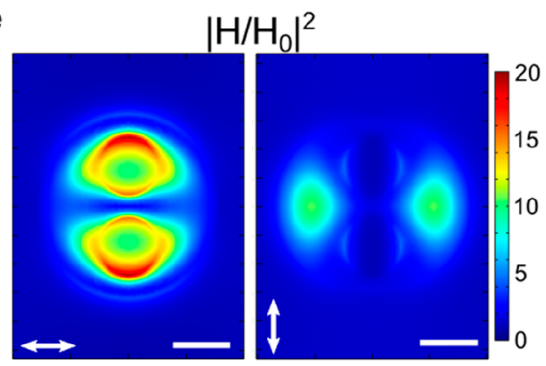

g

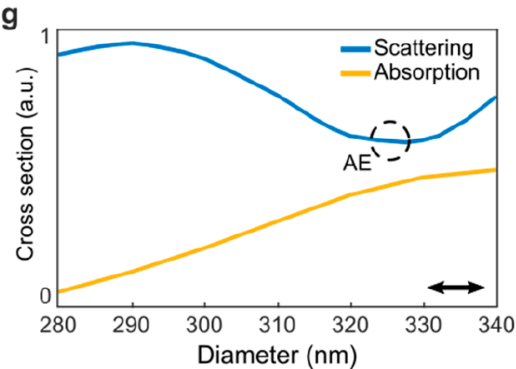

Figure 1. Germanium nanoantennas sample. (a) SEM image of the fabricated array of nanoantennas. The external diameter $D$ ranges from 260 to $350 \mathrm{~nm}$. Scale bar: $1 \mu \mathrm{m}$. The inset shows a magnified SEM image of a single nanodisk; each hole has a diameter of $100 \mathrm{~nm}$. Scale bar: $200 \mathrm{~nm}$. (b) Tapping mode AFM image of a single nanoantenna. (c) Schematic representation of the anapole excitation (AE) in a germanium nanodisk. Linearly polarized light impinges from below in the $Z$-direction and excites the sample at resonance; electric and magnetic fields build up inside the nanoantenna. (d,e) Calculated electric (d) and magnetic (e) near-field field intensity distributions for horizontal and vertical polarizations, as indicated by the arrows, computed at midheight of a nanoantenna of $D=320 \mathrm{~nm} . E_{0}$ and $H_{0}$ are the amplitude of the electric and magnetic incident fields, respectively. Scale bar: $100 \mathrm{~nm}$. (f) Experimental optical scattering of nanoantennas of different diameters taken at 800 nm wavelength illumination. (g) Corresponding simulated scattering and absorption cross sections computed at 850 nm wavelength to better describe the experimental results (see main text).

by a light beam. ${ }^{17-22}$ However, while the distribution of the electric and magnetic near fields around a dielectric nanoantenna has been characterized theoretically and experimentally, ${ }^{23}$ and even the optical forces produced on dielectric particles have been measured, ${ }^{24}$ the associated near-field forces they would induce have not yet been measured, although they have been analyzed in theory. ${ }^{22,25}$ In general, the quantitative measurement of optical near-field forces is of great interest for applications related to near-field trapping of nanoparticles and molecules in nanometric volumes, ${ }^{26,27}$ such as biosensing ${ }^{28,29}$ and lab-on-a-chip experiments. ${ }^{30,31}$ In addition, the existence of forces of both magnetic and electrical origin in nanostructured dielectrics allows envisioning some type of sorting of particles and molecules in the near field via specific coupling depending on their electric or magnetic nature, in an analogous way that electric or magnetic dipoles can probe the local density of states of the optical electric or magnetic near fields, respectively. ${ }^{32}$ Furthermore, when designing a nanophotonic hybrid system, there will be a differential local stress to consider over a material placed in the near-field region of the dielectric component, ${ }^{33,34}$ since the electric and magnetic optical fields concentrate in different locations around the dielectric. Knowing that even small movements can translate into large changes in the optical properties of a hybrid system, ${ }^{35}$ the nature, spatial distribution and strength of the near-field forces are of great importance for the construction of nanophotonic devices.

To maximize sensitivity in optical force detection, goldcoated AFM tips have been usually employed in PiFM configurations, ${ }^{13,15,16}$ considerably enhancing the optical electric field at the apex of the tip. However, such a local probe is poorly sensitive to magnetic near fields. Alternatively, a configuration using a dielectric AFM tip, which can be electrically and magnetically polarized by light, has been proposed theoretically ${ }^{36}$ and used in an experiment only to minimize the disturbance on the spectroscopic response of a plasmonic sample. ${ }^{14}$ Here, we successfully implement a PiFM tool that uses a dielectric silicon tip to image both electric and magnetic forces in individual germanium nanoantennas under illumination, thus revealing the spatial distribution of the induced optical forces and the confinement of electric and magnetic fields. The magnitude of the measured forces ranges in the tens of piconewtons, comparable with a variety of other forces such as Casimir $^{37}$ and van der Waals ${ }^{38}$ forces, intramolecular forces engaged in unfolding proteins, ${ }^{39}$ or exerted by individual molecular motors. ${ }^{40}$ Numerical simu- 
lations using finite-difference time-domain methods show very good agreement with the experiment. Our results bring new nanoscale spatial information on the optical forces acting in a single dielectric nanoresonator, revealing a dominant magnetic contribution.

Germanium nanoantennas of $80 \mathrm{~nm}$ height were fabricated by means of thermal evaporation and electron beam lithography on a glass substrate (see Methods for fabrication details). The nanostructures were designed to have a cylindrical shape with two holes along one of its diameters, as shown in the scanning electron microscope (SEM) image of Figure 1a. Throughout the sample, the external diameter $(D)$ of the nanostructures was varied between 260 and $350 \mathrm{~nm}$, while the diameter of the holes was kept fixed at $100 \mathrm{~nm}$. A pitch of $3 \mu \mathrm{m}$ was chosen to avoid optical coupling between adjacent nanoantennas.

The sample was characterized in situ with a combined homemade optical-AFM microscope, capable of achieving wide-field and confocal optical microscopy simultaneously with contact or tapping mode of atomic force imaging. Figure $1 \mathrm{~b}$ displays an AFM image of a nanoantenna taken in tapping mode, showing the dimensions of the nanodisk as well as the ability of the technique to perform a precise inspection of the internal holes. To identify the resonant modes of the germanium nanostructure, shown schematically in Figure 1c, we carried out optical scattering measurements and plane wave finite-difference time domain simulations of scattering and absorption cross sections (see Methods for simulation details). Figure 1f,g exhibits the obtained experimental and theoretical results for nanodisks of different diameters, respectively. The experimental illumination wavelength corresponds to $800 \mathrm{~nm}$, whereas the wavelength in the simulation was set to $850 \mathrm{~nm}$ to better reproduce the measurement (refer to the Supporting Information S3 for the simulation of the scattering spectrum). Similar discrepancies in the resonant wavelength have been previously observed in studies of dielectric nanoantennas and have been ascribed to geometrical imperfections of the fabricated structures. ${ }^{41}$ A relative scattering minimum is observed for a disk diameter of $320 \mathrm{~nm}$, which is assigned to the anapole excitation $(\mathrm{AE})$, as deduced from the corresponding electric and magnetic field intensity distributions shown in Figure $1 \mathrm{~d}, \mathrm{e}$, respectively. The $\mathrm{AE}$ is a nonradiative state, product of destructive interference between electrical and toroidal dipole modes, ${ }^{42,43}$ and has been recently observed in simple structures such as nanodisks of silicon, ${ }^{42}$ germanium, ${ }^{41}$ and titanium dioxide. ${ }^{44}$ At the $\mathrm{AE}$ condition, displacement currents circulate in opposite directions on the sides of the disk, with a circular magnetic field that points in the perpendicular direction at the top surface of the disk, as illustrated in Figure 1c. For polarizations both horizontal (i.e., in the direction perpendicular to the line connecting the holes) and vertical (parallel to the line connecting the holes), the magnetic field is highly confined inside the nanoantenna ${ }^{45}$ with enhancement factors that are significantly larger compared to the electric field, which is located mainly at the external edges of the nanoantenna.

To perform the optical force measurements, we modified our optical-AFM system to include PiFM capabilities, reaching the final form schematized in Figure 2a. In the modified setup, the sample is optically excited with a CW Ti:sapphire laser at $800 \mathrm{~nm}$, and its optical response is measured through the induced forces on a silicon AFM tip (Olympus OMCLAC240TS, nominal tip radius $7 \mathrm{~nm}$ ), set to scan over the a

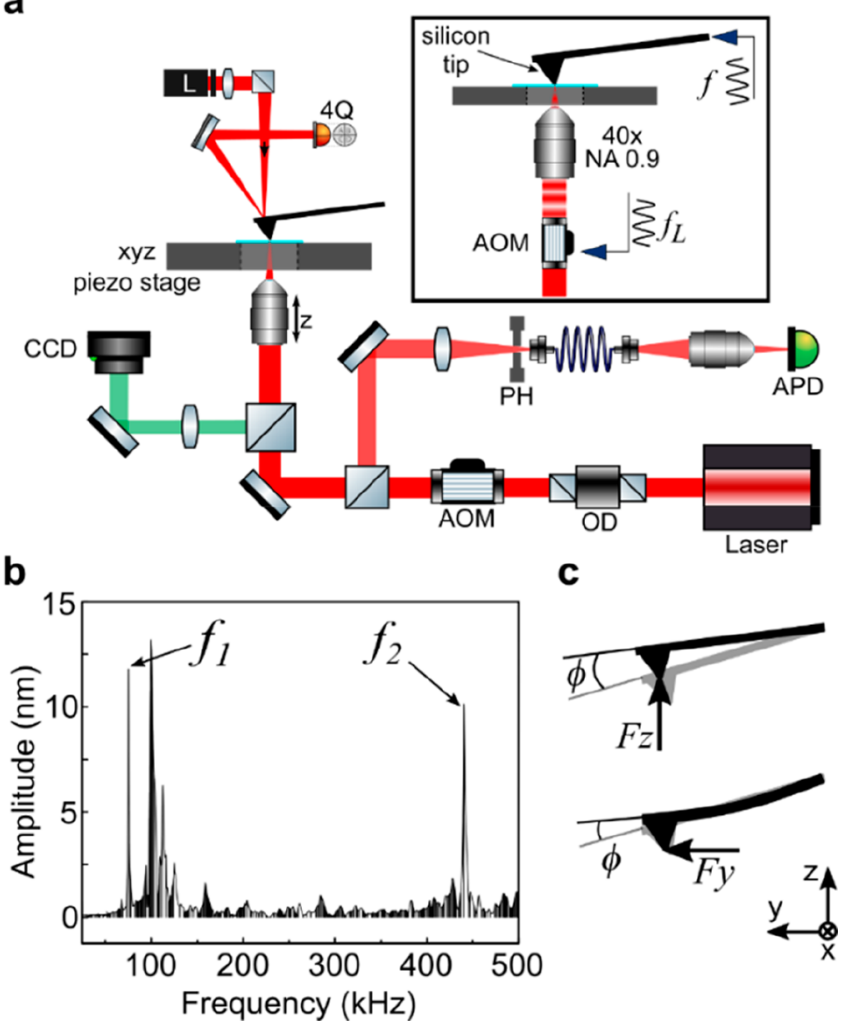

Figure 2. Photoinduced force microscopy (PiFM) scheme. (a) Schematic of the combined optical/atomic force microscope used for the experimental measurements. OD, optical diode; AOM, acoustooptic modulator; PH, pinhole; APD, avalanche photodiode; $4 \mathrm{Q}$, fourquadrant AFM head detection. A detailed view in the square panel shows the configuration used for PiFM operation with simultaneous AFM topography and optical force detection. Two lock-in amplifiers record the voltage signal at the tapping modulation frequency, $f$, and the laser light modulation frequency, $f_{\mathrm{L}}$, respectively. (b) Frequency spectrum of mechanical resonances of the AFM cantilever. $f_{1}$ and $f_{2}$ denote the first and second mechanical resonances, respectively. The peaks around $100 \mathrm{kHz}$ are not characteristic of the cantilever and depend on the coupling of the AFM tip substrate to the holder. (c) Schematics of the deflection of the cantilever when exerting forces on the tip in $Z$ - and $Y$-directions (see Supporting Information S2 for details).

nanoantenna. We used a silicon probe instead of a conventional plasmonic tip so as not to mask the effects of the optical magnetism present in these nanostructures. To register simultaneous topography and photoinduced force measurements, we took advantage of the multiple mechanical resonances supported by the AFM cantilever, ${ }^{46,47}$ which can be seen in the frequency sweep shown in Figure $2 b$, recorded with the silicon tip far from the sample. We found two main resonances at $f_{1} \cong 75 \mathrm{kHz}$ and $f_{2} \cong 440 \mathrm{kHz}$, with corresponding nominal force constants of $k_{1} \cong 2 \mathrm{~N} / \mathrm{m}$ and $k_{2} \cong 80 \mathrm{~N} / \mathrm{m}$, respectively. We note that this observation matches that expected for a rectangular cantilever, where the second resonance is calculated to be close to the sixth harmonic of the fundamental frequency. ${ }^{47}$ In this experimental scheme, by setting the AFM tapping mode at the oscillating frequency $f \sim f_{1}$ and modulating the intensity of the laser beam at $f_{\mathrm{L}}=f_{2}$, we could register both topography and amplified photoinduced effects at independent mechanical resonances using two lock-in amplifiers. Before each set of measurements, 
a

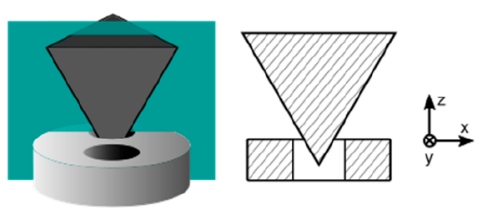

b

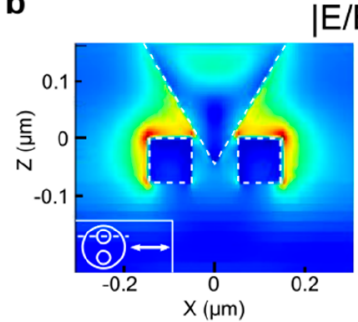

$\left|E / E_{0}\right|^{2}$
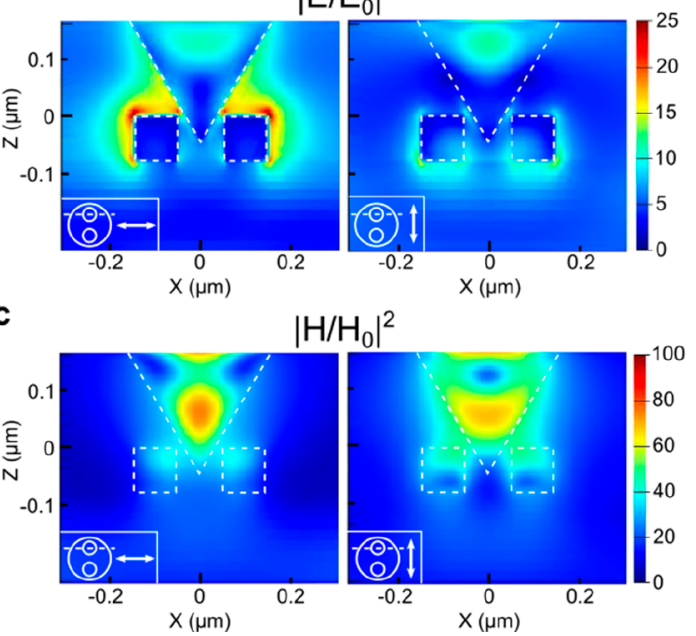

d

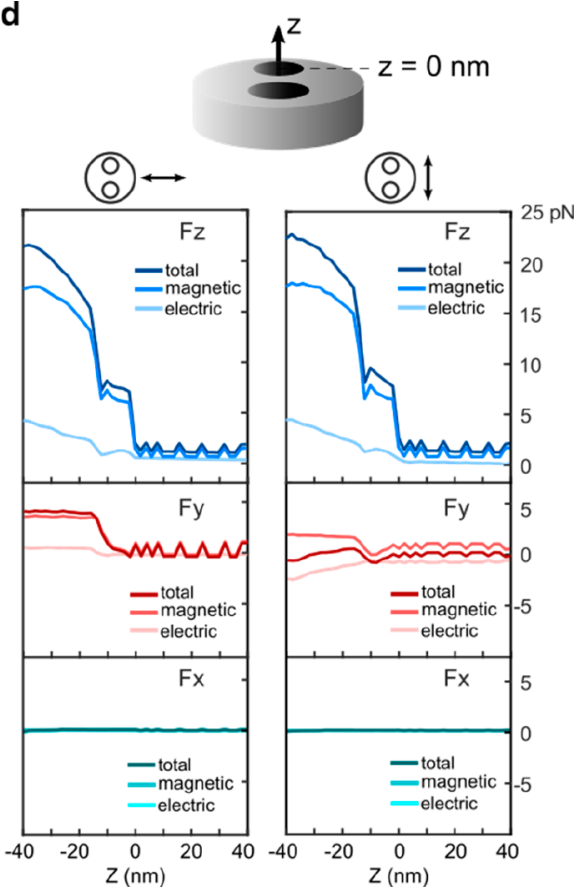

Figure 3. Field and force simulations of the nanoantenna-tip coupled system. (a) Schematic illustration of the $X Z$ cut (in green) taken for the simulations of the fields. The tip is positioned at one of the holes with its apex at midheight inside the nanoantenna. $(b, c)$ Simulated electric $(b)$ and magnetic $(c)$ field intensity distributions of the coupled nanoantenna-tip system described in (a) for horizontal and vertical polarizations. (d) Calculated components of the optically induced electric and magnetic forces on the silicon tip for different positions along the hole $Z$-axis at a light fluence of $1 \mathrm{~mW} / \mu \mathrm{m}^{2}$ for both incident polarizations. In the abscissa, zero refers to the top surface of the antenna whereas negative (positive) means inside (outside) the nanoantenna.

precise values of $k_{1}$ and $k_{2}$ were determined using the usual thermal tune method. ${ }^{48}$

In most of the PiFM configurations found in the literature, sideband detection (i.e., $f_{\mathrm{L}}$ around but not equal to $f_{\text {Cantilever }}$ ) is performed to remove the long-range force background. ${ }^{13,15,16}$ However, we note that sideband detection does not eliminate photothermal contributions and, furthermore, due to the small magnitude of the forces involved it may provide poor sensitivity performance. In our experiment, we chose to execute measurements at maximum sensitivity $\left(f_{\mathrm{L}}=f_{2}\right)$ with careful subtraction of the long-range force background and using a phase-sensitive detection of the lock-in signal at $f_{\mathrm{L}}$ to eliminate the influence of photothermal effects. Indeed, while photoinduced forces are in quadrature with the laser modulation for the mechanical oscillator at resonance, ${ }^{49}$ forces of thermal origin will show a certain phase shift that is dependent on the modulation frequency. ${ }^{50}$ In particular, for high enough frequencies such as those used in our measurements, the photothermal contributions will appear in phase with the modulation. Therefore, the lock-in signals in phase with the laser modulation signal were filtered out from our PiFM measurements (see Supporting Information S1 for a more detailed analysis and explanation). It is also important to mention that our setup was able to detect forces not only in the $Z$-direction (perpendicular to the sample) as usual in any force microscope, but also in the $Y$-direction (along the cantilever long axis), as illustrated in Figure 2c, a component which is not commonly present in topography or friction experiments. Both force components produce an angular deflection on the cantilever which is detectable by the AFM head with a force sensitivity ratio estimated to be $1: 8(Y: Z)$ (see analytical calculation included in the Supporting
Information S2). A voltage/force experimental calibration curve was used to convert lock-in voltage values to force values (see Supporting Information S1).

Given that optical force measurements involve placing the silicon AFM tip in the vicinity of the germanium nanoantenna, it is important to analyze first how the fields would develop in the coupled system. We start by considering the situation where the tip is positioned at the center of one of the holes of the resonant $D=320 \mathrm{~nm}$ nanodisk (see schematic in Figure $3 a$ ), as shown in Figure 3 b,c. For both horizontal and vertical polarizations, we observe that the electric and magnetic fields locate inside and around the nanoantenna-tip system. In particular, we find that the magnetic field concentrates efficiently inside the tip, while the electric field seems to spill out to the borders of the nanoantenna, thus validating the choice of the silicon probe for magnetic force measurements. By comparing the magnetic field maps in Figure 1 (without tip) with those in Figure 3, we observe that magnetic field values in the nanoantenna are of the same order in both cases. We also encounter that the magnitude of the magnetic field inside the tip, which is significantly higher than inside the nanoantenna, is similar to that computed when considering the tip in the absence of the nanoantenna, as shown in the Supporting Information S4. However, as the incident magnetic field is polarized within the $X Y$ plane, the magnetic moment it induces on the tip will not participate in the detection of forces in the (perpendicular) $Z$-direction. The $Z$-direction is the predominant direction for the field produced by the nanodisk inside the hole, which in turn induces a dipolar moment on the tip in the same direction. Therefore, the experiment is expected to mainly probe the interaction between the nanoantenna and its induced dipolar moment on the tip. 

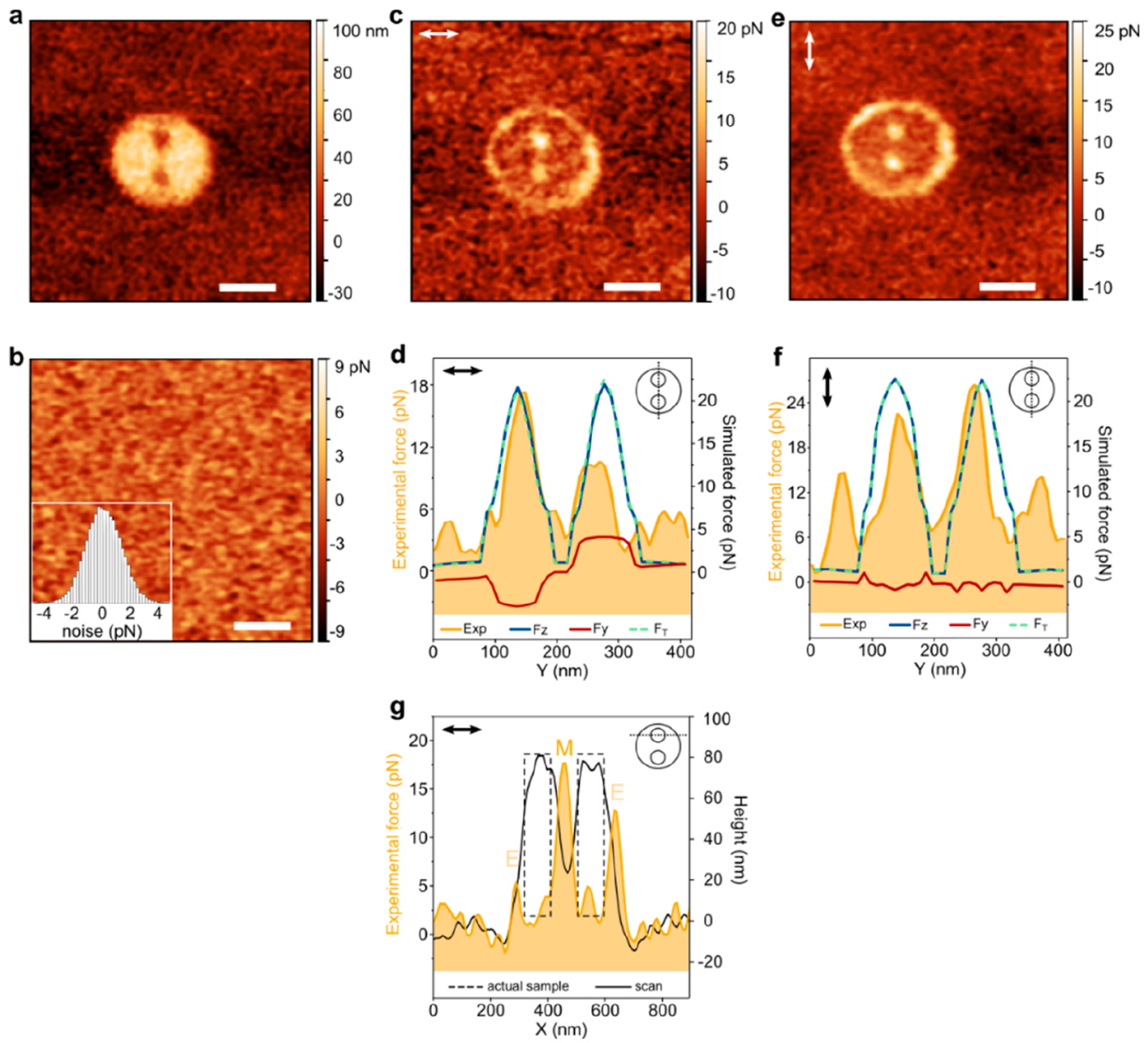

Figure 4. PiFM measurements on a germanium nanoantenna excited at resonance. (a) AFM topography image of a $D=320 \mathrm{~nm}$ germanium nanoantenna. Scale bar: $200 \mathrm{~nm}$. (b) Control PiFM image taken with no illumination in the same region as in (a). The inset shows the corresponding noise histogram. (c,e) PiFM images of the nanoantenna under $1 \mathrm{~mW} / \mu \mathrm{m}^{2}$ illumination fluence with horizontal (c) and vertical (e) polarizations. Color bar scale in piconewtons is obtained after a voltage/force calibration, as shown in Supporting Information S1. (d,f) Experimental force profile along the line crossing the two holes (golden curve) for horizontal (d) and vertical (f) polarizations. Simulated forces in $Y$ - (red) and Z- (blue) directions are included for comparison. The total force $F_{\mathrm{T}}$ is shown in dashed green line, calculated as $F_{\mathrm{T}}=F_{z}+F_{y} / 8$ according to the sensitivity of the experiment. (g) Experimental force (golden curve) and topography (black solid line) profiles taken in $X$-direction crossing one of the holes for horizontal polarization. The dashed line represents the actual topography of the sample. M, magnetic; E, electric (see main text).

Next, we computed numerically the magnitude and character of the optical force that would act on the silicon tip in a PiFM measurement under the specified experimental conditions. First, we evaluated how the force would change as the tip enters the nanoantenna through one of the holes, which corresponds to the path crossing the largest field gradients. To that end, we calculated the induced electric and magnetic forces in each of the three directions for different positions of the tip along the hole $Z$-axis, considering plane wave illumination with horizontal and vertical polarizations at a fluence of $1 \mathrm{~mW} / \mu \mathrm{m}^{2}$. The results are shown in Figure $3 \mathrm{~d}$, where positive and negative $Z$ values indicate positions of the tip outside and inside the hole, respectively (i.e., $Z=0 \mathrm{~nm}$ corresponds to the top surface of the disk). We find that the net force is mainly magnetic in nature and that it occurs mostly in the $Z$-direction, although a small $Y$-component is also present. For the two polarizations, maximum force values reach a few tens of piconewtons at midheight of the disk and decrease to negligible levels when the tip exits the hole. Noticeably, while the tip clearly detects how the force changes with the $Z$-position, it fails to distinguish between horizontal and vertical polarizations. Even though the magnetic field inside the hole is greater for horizontal polarization incidence (Figure 1e), the tip still strongly couples to the fields outside the hole for the vertical polarization (Figure 3c), compensating the difference. Regarding the force in the $X$-direction, it is observed to be zero due to the symmetry of the problem.

Figure 4 summarizes the results from the PiFM measurements performed on a $320 \mathrm{~nm}$ diameter germanium nanoantenna at an illumination fluence of $1 \mathrm{~mW} / \mu \mathrm{m}^{2}$ (see representative AFM topography map in Figure 4a). A PiFM control image taken with no illumination, as shown in Figure $4 \mathrm{~b}$, was used to estimate the detection limit of our experiment. As can be seen from the noise histogram in the inset of the figure, we find this limit to be of the order of $2 \mathrm{pN}$. Figure $4 \mathrm{c}, \mathrm{e}$ presents the PiFM images acquired for horizontal and vertical incident polarizations, respectively. The $1 \times 1 \mu \mathrm{m}^{2}$ images contain $128 \times 128$ pixels, registered at $156 \mathrm{~ms}$ integration time per pixel. In both cases, strong force responses are registered at hole regions and edges of the nanoantenna, while the measured signal on its top surface and outside antenna regions is nearly negligible. Figure 4d,f shows optical force traces along the line 

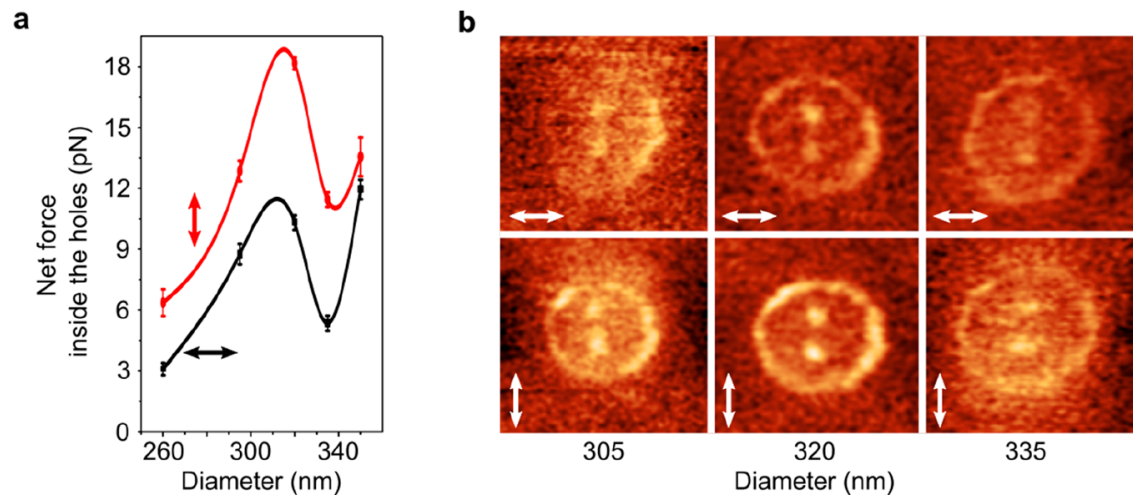

Figure 5. Resonant behavior detected by PiFM. (a) Experimental optical force amplitude inside the two holes (averaged) of the nanoantenna for different disk diameters at $1 \mathrm{~mW} / \mu \mathrm{m}^{2}$ illumination fluence for horizontal and vertical polarizations. (b) Corresponding PiFM images for representative sizes around $D=320 \mathrm{~nm}$.

crossing the disk holes in Figure 4c,e, respectively. The graphs also include corresponding numerical simulations computed by tip-scanning over the antenna's top surface and inside hole regions (see Supporting Information S3 for simulation force maps). A very good agreement between experiment and theory is observed, with simulated and measured force values matching within a factor $<2$. Given the low magnitude of the calculated $Y$-component of the forces and the reduced force sensitivity of the measurement in that direction, we deduce that the experimental signal corresponds essentially to the $Z$ component of the optical force, which is $80 \%$ of magnetic character, according to the numerical simulations. Figure $4 \mathrm{~g}$ shows the optical force trace taken along a horizontal line crossing one of the holes for horizontal polarization illumination. The actual profile of the sample is shown in dashed line for reference, whereas the topography scan line is marked as a solid line; the photoinduced force signal is shown in golden. The finite tip size affects the lateral resolution in such a way that the topography profile results in a convolution between the tip and the sample shapes. For the photoinduced force, we see a central peak that corresponds to the (mainly) magnetic force inside the hole, and two lateral smaller peaks that correspond to the circumference seen in Figure $4 \mathrm{c}$. By an inspection on the simulations, we can assign these latter peaks to forces of electrical origin that exist just outside the nanoantenna.

To further verify the resonant behavior of the chosen germanium nanoantenna, we acquired PiFM images of nanoantennas of different sizes around $D=320 \mathrm{~nm}$, as shown in Figure 5. Figure 5a exhibits the dependence on $D$ of the measured force signal inside the holes of the nanodisk, while Figure 5b displays PiFM images for three representative cases. For both illumination polarizations, we observe that the detected force reduces in magnitude up to 3-4 times for diameters just above and below $320 \mathrm{~nm}$, confirming the presence of a resonance effect at $D=320 \mathrm{~nm}$. Consistent with this, we also find that the defined force pattern observed for $D$ $=320 \mathrm{~nm}$ fades away when increasing or decreasing $D$, due to the reduced field confinement.

In summary, we have shown for the first time to the best of our knowledge that a photoinduced force microscopy configuration using a dielectric silicon tip represents a successful approach to measure optical forces of electric and magnetic character at the nanoscale. We demonstrate that an especially designed germanium nanoantenna can induce forces of up to $\sim 20 \mathrm{pN}$ under resonant anapole illumination when the silicon tip access inside the hollow regions of the nanostructure. We found that the force is mostly magnetic in nature, as revealed by numerical simulations. The results of this investigation demonstrate the capability of dielectric nanoantennas of efficiently confining optical magnetic fields, producing magnetic light forces which are comparable in magnitude to the electric forces detected in plasmonic nanoantennas.

\section{METHODS}

Fabrication of Germanium Nanoantennas. Arrays of germanium nanoantennas were fabricated on borosilicate glass substrate via electron beam lithography. A clean substrate was first coated with poly(methyl methacrylate) (950 K A4) ebeam resist and baked at $180{ }^{\circ} \mathrm{C}$ for $5 \mathrm{~min}$. The nanostructures were then defined by an electron beam exposure at $20 \mathrm{keV}$ (Raith eLINE) with a conductive layer of Espacer (Showa Denko), followed by a standard development procedure with isopropanol and methyl isobutyl ketone. Germanium was thermally evaporated at $1.5 \AA / \mathrm{s}$ and carefully removed by MICROPOSIT 1165 as the final lift-off step.

Simulation of Scattering and Absorption Cross Sections. Numerical studies of the electromagnetic responses were performed using a finite-difference time-domain (FDTD) commercial software (Lumerical FDTD Solutions 2019a) with normal incidence through the substrate. The dielectric functions of germanium were extracted from our previous measured ellipsometry results. ${ }^{41,51,52}$ Total-field scattered-field (TFSF) source was used to investigate the scattering and absorption performance of the individual nanoantennas.

\section{ASSOCIATED CONTENT}

\section{Supporting Information}

The Supporting Information is available free of charge at https://pubs.acs.org/doi/10.1021/acs.nanolett.0c03157.

Phase-sensitive detection and photothermal contribution; detection sensitivity of the force components; simulation of the scattering cross section and force maps; field simulations for the tip in the absence of the nanoantenna (PDF) 


\section{AUTHOR INFORMATION}

\section{Corresponding Authors}

Stefan A. Maier - Chair in Hybrid Nanosystems, Nanoinstitute Munich, Faculty of Physics, Ludwig-Maximilians-Universität München, 80539 München, Germany; Department of Physics, Imperial College London, London SW7 2AZ, United Kingdom; Email: stefan.maier@physik.uni-muenchen.de

Gustavo Grinblat - Departamento de Física, FCEN, IFIBA CONICET, Universidad de Buenos Aires, C1428EGA Buenos Aires, Argentina; ๑ orcid.org/0000-0002-1637-9524; Email: grinblat@df.uba.ar

\section{Authors}

Martin Poblet - Departamento de Física, FCEN, IFIBA CONICET, Universidad de Buenos Aires, C1428EGA Buenos Aires, Argentina

Yi Li - School of Microelectronics, MOE Engineering Research Center of Integrated Circuits for Next Generation Communications, Southern University of Science and Technology, Shenzhen 518055, China; 0 orcid.org/00000002-6134-3117

Emiliano Cortés - Chair in Hybrid Nanosystems, Nanoinstitute Munich, Faculty of Physics, Ludwig-Maximilians-Universität München, 80539 München, Germany; ๑ orcid.org/00000001-8248-4165

Andrea V. Bragas - Departamento de Física, FCEN, IFIBA CONICET, Universidad de Buenos Aires, C1428EGA Buenos Aires, Argentina; $\odot$ orcid.org/0000-0003-4482-9059

Complete contact information is available at:

https://pubs.acs.org/10.1021/acs.nanolett.0c03157

\section{Author Contributions}

M.P., G.G., and A.V.B. designed the experiments and M.P. implemented the experimental techniques and measurements. Y.L. fabricated the samples and performed all the simulations. S.A.M., A.V.B., G.G., and E.C. conceived the idea, and E.C., S.A.M., A.V.B., and G.G. lead the project. All authors contributed with discussions and the writing of the manuscript.

\section{Notes}

The authors declare no competing financial interest.

\section{ACKNOWLEDGMENTS}

This work was partially supported by PICT 2017-2534, PIP 112201301 00619, UBACyT Proyecto 20020170100432BA. E.C and S.A.M. acknowledge funding and support from the Deutsche Forschungsgemeinschaft (DFG, German Research Foundation) under Germany's Excellence Strategy - EXC 2089/1 - 390776260, the Bavarian program Solar Energies Go Hybrid (SolTech), and the Center for NanoScience (CeNS). S.A.M. further acknowledge funding from the EPSRC (EP/ P033369/1 and EP/M013812/1) and the Lee - Lucas Chair in Physics. E.C. acknowledges funding of the European Commission through the ERC Starting Grant CATALIGHT (802989). Y.L. acknowledges funding received from the Natural Science Foundation of Guangdong Province (2019A1515111136).

\section{REFERENCES}

(1) Ashkin, A.; Dziedzic, J. M.; Bjorkholm, J. E.; Chu, S. Observation of a Single-Beam Gradient Force Optical Trap for Dielectric Particles. Opt. Angular Momentum 1986, 11 (5), 196-198.

(2) Grier, D. G. A Revolution in Optical Manipulation. Nature 2003, $424,810-816$.
(3) Urban, A. S.; Lutich, A. A.; Stefani, F. D.; Feldmann, J. Laser Printing Single Gold Nanoparticles. Nano Lett. 2010, 10 (12), 47944798.

(4) Gargiulo, J.; Violi, I. L.; Cerrota, S.; Chvátal, L.; Cortés, E.; Perassi, E. M.; Diaz, F.; Zemánek, P.; Stefani, F. D. Accuracy and Mechanistic Details of Optical Printing of Single $\mathrm{Au}$ and $\mathrm{Ag}$ Nanoparticles. ACS Nano 2017, 11 (10), 9678-9688.

(5) Leibfried, D.; Blatt, R.; Monroe, C.; Wineland, D. Quantum Dynamics of Single Trapped Ions. Rev. Mod. Phys. 2003, 75 (1), 281324.

(6) Kippenberg, T. J.; Vahala, K. J. Cavity Optomechanics: BackAction at the Mesoscale. Science 2008, 321 (5893), 1172-1176.

(7) Aspelmeyer, M.; Kippenberg, T. J.; Marquardt, F. Cavity Optomechanics. Rev. Mod. Phys. 2014, 86 (4), 1391-1452.

(8) Yu, W.; Jiang, W. C.; Lin, Q.; Lu, T. Cavity Optomechanical Spring Sensing of Single Molecules. Nat. Commun. 2016, 7, 12311.

(9) Schmid, M.; Andrae, P.; Manley, P. Plasmonic and Photonic Scattering and near Fields of Nanoparticles. Nanoscale Res. Lett. 2014, 9 (1), 1-11.

(10) Burresi, M.; Van Oosten, D.; Kampfrath, T.; Schoenmaker, H.; Heideman, R.; Leinse, A.; Kuipers, L. Probing the Magnetic Field of Light at Optical Frequencies. Science 2009, 326 (5952), 550-553.

(11) le Feber, B.; Sipe, J. E.; Wulf, M.; Kuipers, L.; Rotenberg, N. A Full Vectorial Mapping of Nanophotonic Light Fields. Light: Sci. Appl. 2019, 8 (1), 1-7.

(12) Zaza, C.; Violi, I. L.; Gargiulo, J.; Chiarelli, G.; Schumacher, L.; Jakobi, J.; Olmos-Trigo, J.; Cortes, E.; König, M.; Barcikowski, S.; Schlücker, S.; Sáenz, J. J.; Maier, S. A.; Stefani, F. D. Size-Selective Optical Printing of Silicon Nanoparticles through Their Dipolar Magnetic Resonance. ACS Photonics 2019, 6 (4), 815-822.

(13) Tumkur, T. U.; Yang, X.; Cerjan, B.; Halas, N. J.; Nordlander, P.; Thomann, I. Photoinduced Force Mapping of Plasmonic Nanostructures. Nano Lett. 2016, 16 (12), 7942-7949.

(14) Tumkur, T.; Yang, X.; Zhang, C.; Yang, J.; Zhang, Y.; Naik, G. V.; Nordlander, P.; Halas, N. J. Wavelength-Dependent Optical Force Imaging of Bimetallic Al-Au Heterodimers. Nano Lett. 2018, 18 (3), 2040-2046.

(15) Rajapaksa, I.; Uenal, K.; Wickramasinghe, H. K. Image Force Microscopy of Molecular Resonance: A Microscope Principle. Appl. Phys. Lett. 2010, 97 (7), 073121.

(16) Huang, F.; Tamma, V. A.; Mardy, Z.; Burdett, J.; Wickramasinghe, H. K. Imaging Nanoscale Electromagnetic NearField Distributions Using Optical Forces. Sci. Rep. 2015, 5, 1-12.

(17) Nieto-Vesperinas, M.; Sáenz, J. J.; Gómez-Medina, R.; Chantada, L. Optical Forces on Small Magnetodielectric Particles. Opt. Express 2010, 18 (11), 11428-11443.

(18) Chaumet, P. C.; Rahmani, A. Electromagnetic Force and Torque on Magnetic and Negative-Index Scatterers. Opt. Express 2009, 17 (4), 2224-2234.

(19) Nieto-Vesperinas, M.; Gomez-Medina, R.; Saenz, J. J. AngleSuppressed Scattering and Optical Forces on Submicrometer Dielectric Particles. J. Opt. Soc. Am. A 2011, 28 (1), 54.

(20) Lester, M.; Arias-González, J. R.; Nieto-Vesperinas, M. Fundamentals and Model of Photonic-Force Microscopy. Opt. Lett. 2001, 26 (10), 707-709.

(21) Bliokh, K. Y.; Bekshaev, A. Y.; Nori, F. Extraordinary Momentum and Spin in Evanescent Waves. Nat. Commun. 2014, 5, 3300.

(22) Li, H.; Cao, Y.; Zhou, L.-M.; Xu, X.; Zhu, T.; Shi, Y.; Qiu, C.W.; Ding, W. Optical Pulling Forces and Their Applications. Adv. Opt. Photonics 2020, 12 (2), 288-366.

(23) Bakker, R. M.; Permyakov, D.; Yu, Y. F.; Markovich, D.; Paniagua-Dominguez, R.; Gonzaga, L.; Samusev, A.; Kivshar, Y.; Luk'yanchuk, B.; Kuznetsov, A. I. Magnetic and Electric Hotspots with Silicon Nanodimers. Nano Lett. 2015, 15 (3), 2137-2142.

(24) Antognozzi, M.; Bermingham, C. R.; Harniman, R. L.; Simpson, S.; Senior, J.; Hayward, R.; Hoerber, H.; Dennis, M. R.; Bekshaev, A. Y.; Bliokh, K. Y.; Nori, F. Direct Measurements of the Extraordinary 
Optical Momentum and Transverse Spin-Dependent Force Using a Nano-Cantilever. Nat. Phys. 2016, 12 (April), 731-735.

(25) Gao, D.; Ding, W.; Nieto-Vesperinas, M.; Ding, X.; Rahman, M.; Zhang, T.; Lim, C. T.; Qiu, C. W. Optical Manipulation from the Microscale to the Nanoscale: Fundamentals, Advances and Prospects. Light: Sci. Appl. 2017, 6 (9), e17039.

(26) Righini, M.; Zelenina, A. S.; Girard, C.; Quidant, R. Parallel and Selective Trapping in a Patterned Plasmonic Landscape. Nat. Phys. 2007, 3 (7), 477-480.

(27) Juan, M. L.; Gordon, R.; Pang, Y.; Eftekhari, F.; Quidant, R. Self-Induced Back-Action Optical Trapping of Dielectric Nanoparticles. Nat. Phys. 2009, 5 (12), 915-919.

(28) Righini, M.; Ghenuche, P.; Cherukulappurath, S.; Myroshnychenko, V.; Garcia de Abajo, F. J.; Quidant, R. NanoOptical Trapping of Rayleigh Particles and Escherichia Coli Bacteria with Resonant Optical Antennas. Nano Lett. 2009, 9 (10), 33873391.

(29) Koya, A. N.; Cunha, J.; Guo, T. L.; Toma, A.; Garoli, D.; Wang, T.; Juodkazis, S.; Cojoc, D.; Proietti Zaccaria, R. Novel Plasmonic Nanocavities for Optical Trapping-Assisted Biosensing Applications. Adv. Opt. Mater. 2020, 8 (7), 1901481.

(30) Huang, L.; Maerkl, S. J.; Martin, O. J. F. Integration of Plasmonic Trapping in a Microfluidic Environment. Opt. Express 2009, 17 (8), 6018-6024.

(31) Huang, J. S.; Yang, Y. T. Origin and Future of Plasmonic Optical Tweezers. Nanomaterials 2015, 5 (2), 1048-1065.

(32) Taminiau, T. H.; Karaveli, S.; Van Hulst, N. F.; Zia, R. Quantifying the Magnetic Nature of Light Emission. Nat. Commun. 2012, 3, 979.

(33) Zhang, J.; Yasuda, H.; MacDonald, K. F.; Zheludev, N. I. Optical Gecko Toe: Optically Controlled Attractive near-Field Forces between Plasmonic Metamaterials and Dielectric or Metal Surfaces. Phys. Rev. B: Condens. Matter Mater. Phys. 2012, 85, 205123.

(34) Li, X.; Zhu, J.; Wei, B. Hybrid Nanostructures of Metal/TwoDimensional Nanomaterials for Plasmon-Enhanced Applications. Chem. Soc. Rev. 2016, 45 (11), 3145-3187.

(35) Zheludev, N. I.; Plum, E. Reconfigurable Nanomechanical Photonic Metamaterials. Nat. Nanotechnol. 2016, 11 (1), 16-22.

(36) Guclu, C.; Tamma, V. A.; Wickramasinghe, H. K.; Capolino, F. Photoinduced Magnetic Force between Nanostructures. Phys. Rev. B: Condens. Matter Mater. Phys. 2015, 92 (23), 235111.

(37) Decca, R. S.; López, D.; Fischbach, E.; Krause, D. E. Measurement of the Casimir Force between Dissimilar Metals. Phys. Rev. Lett. 2003, 91 (5), 3-6.

(38) Kawai, S.; Foster, A. S.; Björkman, T.; Nowakowska, S.; Björk, J.; Canova, F. F.; Gade, L. H.; Jung, T. A.; Meyer, E. Van Der Waals Interactions and the Limits of Isolated Atom Models at Interfaces. Nat. Commun. 2016, 7, 11559.

(39) Bustamante, C. Of Torques, Forces, and Protein Machines. Protein Sci. 2004, 13 (11), 3061-3065.

(40) Finer, J. T.; Simmons, R. M.; Spudich, J. A. Single Myosin Molecule Mechanics: Piconewton Forces and Nanometre Steps. Nature 1994, 368 (6467), 113-119.

(41) Grinblat, G.; Li, Y.; Nielsen, M. P.; Oulton, R. F.; Maier, S. A. Enhanced Third Harmonic Generation in Single Germanium Nanodisks Excited at the Anapole Mode. Nano Lett. 2016, 16 (7), 4635-4640.

(42) Miroshnichenko, A. E.; Evlyukhin, A. B.; Yu, Y. F.; Bakker, R. M.; Chipouline, A.; Kuznetsov, A. I.; Luk'yanchuk, B.; Chichkov, B. N.; Kivshar, Y. S. Nonradiating Anapole Modes in Dielectric Nanoparticles. Nat. Commun. 2015, 6, 8069.

(43) Monticone, F.; Sounas, D.; Krasnok, A.; Alù, A. Can a Nonradiating Mode Be Externally Excited? Nonscattering States versus Embedded Eigenstates. ACS Photonics 2019, 6 (12), 31083114.

(44) Hüttenhofer, L.; Eckmann, F.; Lauri, A.; Cambiasso, J.; Pensa, E.; Li, Y.; Cortés, E.; Sharp, I. D.; Maier, S. A. Anapole Excitations in Oxygen-Vacancy-Rich TiO2- $\mathrm{x}$ Nanoresonators: Tuning the Absorp- tion for Photocatalysis in the Visible Spectrum. ACS Nano 2020, 14 (2), 2456-2464.

(45) Li, J.; Verellen, N.; Van Dorpe, P. Enhancing Magnetic Dipole Emission by a Nano-Doughnut-Shaped Silicon Disk. ACS Photonics 2017, 4 (8), 1893-1898.

(46) Lozano, J. R.; Garcia, R. Theory of Multifrequency Atomic Force Microscopy. Phys. Rev. Lett. 2008, 100 (7), 8-11.

(47) Garcia, R.; Herruzo, E. T. The Emergence of Multifrequency Force Microscopy. Nat. Nanotechnol. 2012, 7 (4), 217-226.

(48) Hutter, J. L.; Bechhoefer, J. Calibration of Atomic-Force Microscope Tips. Rev. Sci. Instrum. 1993, 64 (7), 1868-1873.

(49) Ma, D.; Garrett, J. L.; Munday, J. N. Quantitative Measurement of Radiation Pressure on a Microcantilever in Ambient Environment. Appl. Phys. Lett. 2015, 106 (9), 1901481.

(50) Landi, S. M.; Bragas, A. V.; Coy, J. A.; Martínez, O. E. Avoiding Photothermal Noise in Laser Assisted Scanning Tunneling Microscopy. Ultramicroscopy 1999, 77 (3-4), 207-211.

(51) Grinblat, G.; Li, Y.; Nielsen, M. P.; Oulton, R. F.; Maier, S. A. Efficient Third Harmonic Generation and Nonlinear Sub-Wavelength Imaging at a Higher-Order Anapole Mode in a Single Germanium Nanodisk. ACS Nano 2017, 11 (1), 953-960.

(52) Grinblat, G.; Li, Y.; Nielsen, M. P.; Oulton, R. F.; Maier, S. A. Degenerate Four-Wave Mixing in a Multiresonant Germanium Nanodisk. ACS Photonics 2017, 4 (9), 2144-2149. 